\title{
Content-Based Retrieval for European Image Libraries
}

\author{
C L Bird \\ Applied Science \& Technology Group, IBM UK Laboratories, \\ Hursley Park, Winchester, Hampshire \\ P J Elliott \\ Applied Science \& Technology Group, IBM UK Laboratories, \\ Hursley Park, Winchester, Hampshire \\ P M Hayward \\ Applied Science \& Technology Group, IBM UK Laboratories, \\ Hursley Park, Winchester, Hampshire
}

\begin{abstract}
The principles of content-based image retrieval (CBIR) are now well-established, with a continuing flow of publications exploring various aspects of the technology. Practical applications receive rather less attention and few fully-developed instances have been described. In this paper, we review the remarks made by other authors that led us to believe that it was important to investigate what users expect when using CBIR and how they react to the interface provided. We report the results of observing a number of subjects, with varying experience, while using a CBIR system to search for images. We interpret these observations and obtain insights into the role CBIR might play in meeting the challenge of image retrieval. The work is being carried out as part of the ELISE project (Electronic Library Image Service for Europe), which is partly funded by the European Commission under its Fourth Framework Libraries Programme.
\end{abstract}

\section{Introduction}

The retrieval of images by means of their visual content has been an active research topic for much of this decade, with no sign of flagging interest as we approach the millennium. However, there is presently little evidence of practical applications using the technology, which is disappointing. We distinguish practical embodiments from demonstration systems, of which a number are by now well-known, such as [1-4], although that is far from being a complete list. Furthermore, little attention has been paid to the user requirements and expectations associated with content-based image retrieval. A programme of studies is underway at the University of Northumbria [5] but, aside from that, most reports of work in this area are incidental to other research priorities. We had an opportunity, as part of the ELISE (Electronic Library Image Service for Europe) project, to investigate a user interface design and to collect feedback on patterns of usage, albeit under a restricted set of conditions. The ELISE project and our plan for this work was described as part of a paper presented at the 1998 Challenge of Image Retrieval Workshop [6]. This paper describes the experiment itself, the interface used and the results collected with respect to user responses.

\section{Background}

Content-Based Image Retrieval, now often abbreviated to CBIR, has been characterised in many publications that then describe the associated research activities. In this paper we will not reiterate what has been said before, but rather concentrate on the opportunity offered by the ELISE project to conduct a usability experiment.

\subsection{User Interfaces for Content-Based Image Retrieval}

Amidst a plethora of research reports, little attention has been paid to the user requirements and expectations associated with content-based image retrieval. Even where the work described is set in an application context, there is rarely evidence of a thorough analysis of needs.

Information retrieval specialists have measures such as precision and recall for evaluating queries. There is a 
presumption that definable targets exist and that the measures will indicate how well they have been met. In some cases the same will be true for CBIR, notably where users have clear ideas of what they are looking for. In other situations, typically described by the request: "show me some more images that look like this one", the user's expectations are much less clear. Gupta and Jain say, quite rightly, that "not enough effort has been directed to establishing criteria for evaluating, benchmarking and comparing VIR [Visual Information Retrieval] systems. This lack of effort is in part attributable to the subjective character of the domain." [8]. They go on to consider how to assess "the extent to which a system's query results correspond to user's mental images of what should be retrieved". This is clearly on the right track, but if ways can be found to incorporate those user expectations into the query model, we will be better placed.

Visual forms of relevance feedback have an important role in improving the success rate of searches for individual users. Indeed, relevance feedback for image retrieval is now a significant research topic in its own right: for some examples, see [9-12]. Useful though such studies are, they do not establish beforehand what users are looking for and what their goals are. Hopefully, the work of John Eakins' group at the Institute for Image Data Research will advance our understanding in this latter respect. For an early publication, see [5].

There is, we believe, no pressure to evolve a single style of user interface to meet the general requirements of a range of applications. However, there is a definite need to develop a set of design principles that may be deployed in the majority of situations.

Psarrou et al say: "The need for the development of image indexing and retrieval algorithms tailored to specific domains has been raised by a number of curators and art researchers." [13]. It is only to be expected that requests for specific interfaces will follow. Grout, in concluding a critical review of the potential for CBIR in the Art-Historical domain, expresses the opinion: “... it is evident that the future of the application of image recognition and retrieval techniques to art images relies on working from the particular nature of art images and user requirements upwards. As exciting and scientifically sophisticated as many of the emerging technologies may be, while their user methodologies continue to originate with non-art images it seems likely that they will continue to have a limited utility for the art historical domain. "[14].

It is a characteristic of the current crop of CBIR interfaces that different modes of interaction are adopted. Widespread familiarity with Web browsers has created an expectation that clicking an active area of the visual canvas will result in either more detail or a move to an item of related information. For some CBIR interfaces, clicking an image initiates a new search for more images that look like the one selected. This is commonly described as a query by example. The outcome, far from being related information, may often be a very different set of images, contrary to the user's expectation. It is interesting to note that the AMORE demonstration [4] now uses an adjacent 'Similar' button to initiate a query by example, whereas an earlier version used the image clicking mode.

\subsection{The QBIC System}

We chose IBM QBIC ${ }^{1}$ (Query By Image Content) technology for our CBIR evaluation. QBIC was developed at the IBM Almaden Research Center. Early versions had a variety of features, in particular for colour, texture and shape, and a very flexible interface which was too complex for general users [1]. Some feature queries are more suitable than others for certain categories of image. Queries based on the colour content of images usually work well. Texture features can be used for entire scenes, but it is commonly more appropriate to delineate textured areas and search specifically on those parts of the image. This form of query is not supported in the current release of the code. Shape queries can be problematic, partly because of the difficulty of describing the required shape in a simple enough manner for general users: often the results do not justify the effort. QBIC also has a Colour Layout feature which measures the distribution of colours in the image on a coarse grid, thus combining elements of both colour and shape in the query. It is our belief that this is the most appropriate feature for use in ELISE, especially with coloured 'specimen' images, that is, those with an object or objects against a neutral background. Line drawings, or images that contain little colour, are unlikely to produce useful results with QBIC.

There are two ways of generating a query with QBIC, both of which we provide. The simpler one is to start from an existing image and then request QBIC to find other images which are similar in terms of their colour layout (Query for similar images). The other way is to draw a rough picture of the required image i.e. to define the distribution of colours within it (Draw colour layout). This requires a more complex user interface. Result images are displayed in a form of 'light-box', ranked in order of how closely they match the query. There is no concept of an exact match as there is in text searching: the requested number of images will always be returned. With small image collections, especially those containing a wide variety of images, this may produce surprising results (which can, nevertheless, be useful in leading users to areas of the collection they were previously unaware of).

\footnotetext{
${ }^{1}$ IBM and QBIC are trade marks of International Business Machines Corporation.
} 


\section{Content-Based Image Retrieval for ELISE}

\subsection{Experimental Aims}

It is apparent that there is ample room for research into user interfaces for content-based image retrieval. Given the limited resources within ELISE, we consciously restricted the scope of our use of the QBIC technology to ensure that our experiment was tractable. For the reasons given above, we employed only the Colour Layout feature and chose to concentrate on specimen images, that is those images in which the significant object or objects is or are depicted against a neutral background. We asked providers to bear this in mind when generating their image lists. While this by no means covers all the images in the ELISE collections, it did provide us with a useful subset on which we base our analysis of the results.

Our objective was to assess usability by monitoring user responses. We ran a small number of specific evaluation sessions with an evaluator who recorded the results manually while the subject was using the system. We concentrated on: how users reacted to the CBIR interface; how they used the query options; and their expectations when formulating their queries. Our system automatically recorded data about the query and the results returned. However, the outcomes of individual queries are dependent on both the retrieval technology and the characteristics of the images within the collection. The analysis of such data is beyond the scope of this present paper.

\subsection{Design of the User Interface}

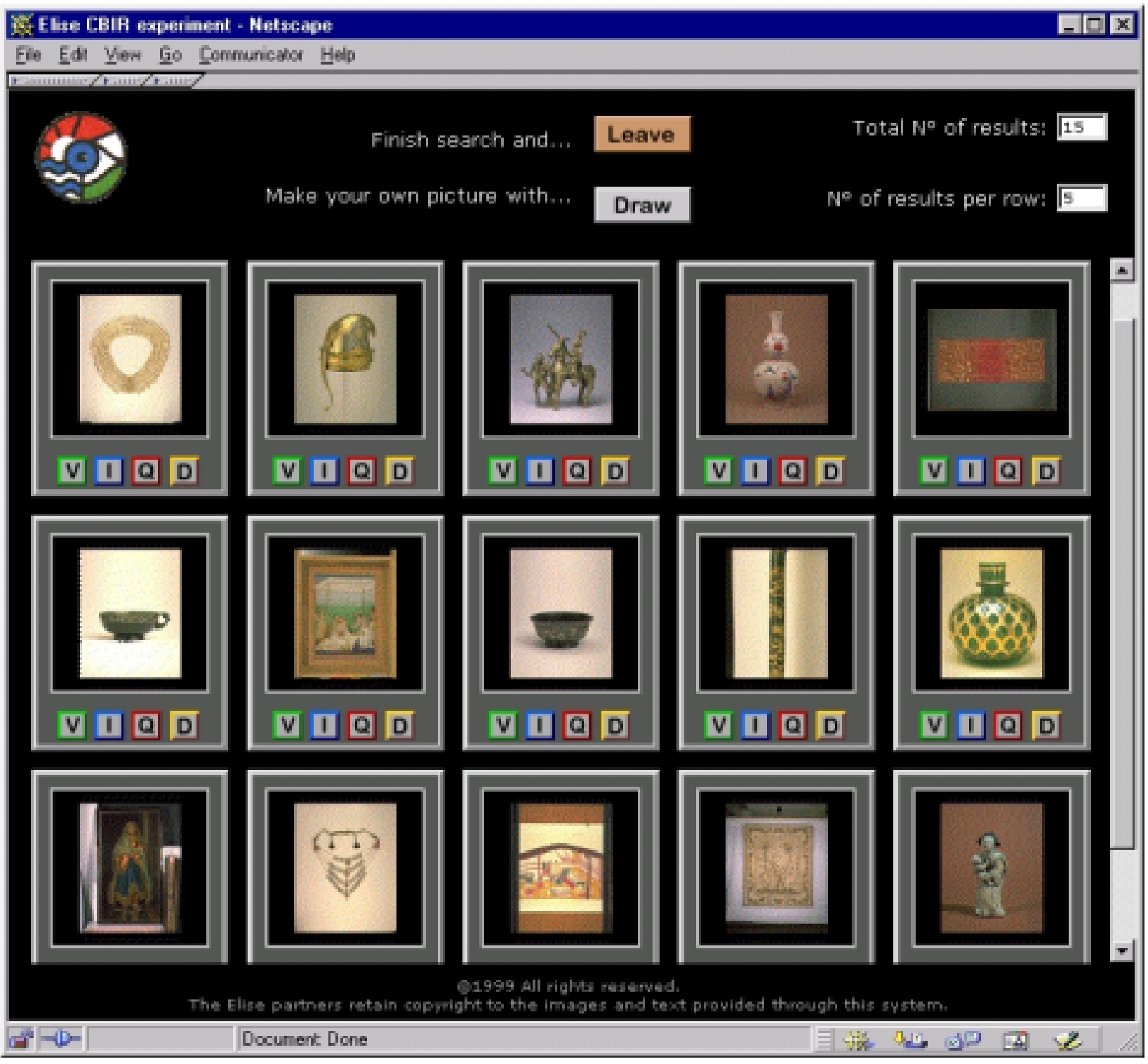

Challenge of Image Retrieval, Newcastle, 1999 
When designing the interface, the chief criterion was simplicity. We made few assumptions about the experience of potential users, beyond their general familiarity with web pages. The controls made available, and their positions within the GUI, were therefore carefully chosen in an attempt to make the task of retrieving images easy and obvious.

In view of the comments made above regarding users' expectations when clicking on an image within a lightbox, we decided to preclude possible confusion resulting from such an action by making the thumbnails themselves inactive. However, all user actions, including these clicks, were monitored. When subjects did select an image in this way, we asked them about their expectations.

The GUI is divided into two parts. In the upper, a small control panel covers actions relating to the application as a whole. As with most other similar interfaces, the main area of the GUI comprises a light-box panel to display thumbnail images from the results of searches. Beneath each image, there are controls for image-specific actions.

Typically, a user wishing to use CBIR from within the ELISE framework has already found an image by means of a text-based search and is interested in finding other, similar, images. The GUI therefore starts with the user's initial image in the top left of the light-box and adds thumbnails of the most similar images from the collection in descending order of similarity. The user may then choose from a number of different options.

If one of the thumbnails in the light-box is of particular interest, clicking on the 'View larger image' button [V] will display the full-size image in a separate window.

Similarly, clicking on the 'Information' button [I] will display the record of textual information associated with the image, in the normal ELISE GUI. (From there, the full range of ELISE functions is available.) Alternatively, the user may wish to continue searching, using any one of the displayed thumbnails as a starting reference. Clicking the 'Query for similar images' button [Q] will start a new query and repopulate the light-box with this 'reference' thumbnail in the top left corner.

If none of the images portrayed are suitable references for initiating a search, the user can choose to 'Draw a colour layout' by clicking on the [Draw] button to start the drawing applet. Alternatively, the user may click on one

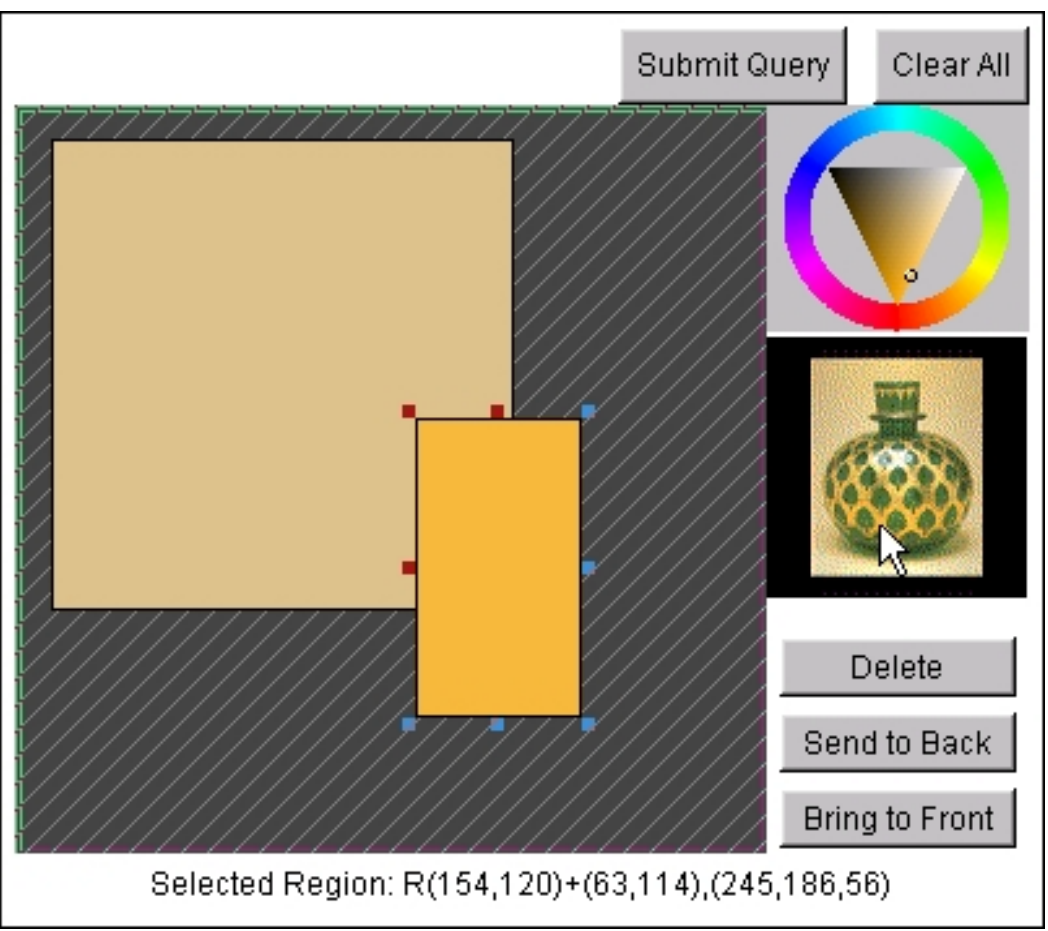
of the [D] buttons, in which case the corresponding thumbnail is imported into the drawing tool and may be used as an alternative to the colour wheel for selecting colours. In the example on the left, two boxes have been created in the drawing area and coloured by selecting from the thumbnail. The user may go on to draw and colour other boxes which can be manipulated using the controls available. When the 'drawing' is complete, the request will be submitted and the GUI light-box filled with those images most closely matching the colour layout depicted. (Areas in the layout not coloured are treated as 'do not mind' by the QBIC Search Engine.) The drawing can be refined and resubmitted as required.

Additional controls are available to allow the user to enter how many images are required in the results and how many thumbnails should be displayed on each row of the light-box.

The user may also leave the CBIR GUI and return to the ELISE interface without exporting a selected image, or group of images, by clicking on the [Leave] button.

To remind users of the function of each button, we provided information popped-up when the mouse pointer was positioned over a button. This is sometimes called 'Hover Help'.

\subsection{Implementation}

The Java Query GUI, as developed by the IBM Almaden Research Center, operates as a stand-alone application, 
whereas it is a prerequisite for ELISE that it is available to users through Internet web pages and Java applets. A Java Query GUI applet is available but experimentation revealed that the same effect could be achieved with fewer overheads in both programming time and in web page download time by using a straightforward HTML approach. One part of the system for which a Java applet is necessary is the 'Draw colour layout' window (see above) and this, being usable in isolation from the rest of the GUI applet, was retained from the QBIC Java code we had obtained from Almaden. The applet was modified by removing some functions not necessary for our purposes and by adding the ability to import a thumbnail image which could be used for selecting colours as described above.

The QBIC Search Engine requires information about images to be stored in QBIC Catalogs. The process of populating the Catalogs began by asking image providers for lists of suitable images. We then created a temporary copy of each image and computed its feature vector. We also produced a thumbnail image, which is stored and subsequently used for display in the light-box.

We had about 2,500 images for this study - mainly from the Victoria \& Albert Museum in London, supplemented with images from the Hunt Museum in Limerick. Other collections in ELISE were unsuitable for CBIR (such as a collection of newspaper images), or not available at the time.

\section{Evaluation}

The rationale for, and aims of, our evaluator-mediated studies were described in the Experimental Aims section. The data collected by the evaluator was structured, involving a mixture of factual recording and questions to capture subjective impressions. An example of the former is whether 'Query for similar images' or 'Draw colour layout' was used.

Prior to beginning the main experiment, we conducted a small pilot study, which revealed a number of points that, for an experiment of this nature, required careful attention:

- The explanatory material needs to be presented in a way that gives sufficient guidance on how to proceed, but is not overwhelming to the extent that it acts as a deterrent in its own right. It is certainly insufficient to rely on verbal instruction alone.

- Users who know little or nothing about the contents of the collection are highly unlikely to bring with them firm ideas of what to look for. Almost all of our subjects were in this category, so providing a selection of target images gave them a purpose. For the small number of subjects performing a repeat evaluation, we asked them to form a mental image of a picture they might be interested in, and to use that as their search target.

- The evaluator needs to be flexible about offering hints and tips. In many cases, it sufficed to remind the subject of a point covered in one of the guidance sheets.

- Evaluation forms must be simple, particularly when used for recording actions which can follow in quick succession, allowing time only for brief notes to be recorded.

\subsection{Methodology}

The format, when the subject was performing the evaluation for the first time, was as follows:

1. Subjects were given a sheet to read, describing the purpose of the evaluation; what we would be recording; and how the evaluation would proceed from there.

2. Basic details about the subjects were recorded, most notably their prior experience of: information retrieval; image retrieval; and content-based image retrieval.

3. Subjects were given a sheet showing twelve target images, ranked approximately in order of difficulty of finding, and asked to choose one of them as their first search target.

4. Subjects were given a sheet giving guidance about the two methods for searching: 'Query for similar images' and 'Draw colour layout'. The information for the latter included a pictorial example of how the first target image could be retrieved by drawing.

5. Subjects were told that they would initially be shown a random set of images from the collection, which might or might not (usually not) help with the search.

6. Subjects were asked to give a verbal indication of their intentions, simply to allow the evaluator to keep track of their actions. The latter were recorded on sheets, each entry was tagged with a code for the action and a screen number ${ }^{2}$. For some actions, such as 'Query for similar images', the reactions of subjects to the resulting set of

\footnotetext{
${ }^{2}$ The screen number was maintained automatically by the system and provided us with the means to relate the action to the data
} 
images were recorded.

7. If subjects became unsure of how to proceed, the evaluator could give hints. These usually took the form of questions intended to encourage the subject to view the problem in a different way. Subjects was never told precisely what to do next. The evaluator noted each occasion on which a hint was provided.

8. When the target image appeared in the light-box, its ranking was noted on the evaluation form.

9. Subjects were then reminded of the options suggested on the description sheet. Those options were listed as:
(a) Look for other, similar, images;
(b) Follow up anything you found interesting;
(c) Choose a fresh target and have another go.

10. The recording of actions continued until the subjects wished to stop, at which point questions were asked about preferences, if any, for drawing a colour layout or querying for similar images. Finally, subjects were asked for any general comments.

Where the subject was performing the evaluation a second time, the search process and the recording of actions remained the same. The guidance sheets were not handed over, but were accessible if required.

\subsection{Results}

Owing to the rather subjective nature of our experiment, we have avoided any form of statistical data presentation. A total of 25 subjects took part in the evaluation, of whom all were familiar with information retrieval to a greater or lesser extent, but only five were familiar with CBIR. Subjects had a variety of background experience, including collection curators, graphic designers, photographers, librarians and IT specialists. Broadly, where a majority of our subjects showed a particular pattern of usage or made a particular comment, we have regarded that as significant. Some of the less prominent observations have also been included for their interest value.

We have divided the results into those related to CBIR in general and those primarily derived from the nature of our user interface. Where both considerations could apply, the presumption is in favour of drawing a lesson for CBIR in general.

\subsubsection{CBIR in general}

- Users accustomed to text-based information retrieval expressed a need for semantic information to assist their searches. They appeared uncomfortable with visual-only methods. Many of the same users also had difficulty moving away from the concept of a 'real' three-dimensional object to that of a two-dimensional representation of the object as areas of colour on a 'canvas'. As one user said, "It is difficult to make the intellectual leap from subject to colour and shape".

- Some users had definite preferences between querying for similar images and drawing a colour layout, while others saw uses for both. Querying is seen to be more useful when exploring; drawing is more effective when the user has a specific goal. With experience, a few users discovered how to use the two in combination, using a query in order to refine the results obtained by drawing.

- Understanding the effect of the image background has a strong dependence on the user's previous experience of working with images, but all were concerned about the effect of background colour on the results. This was generally perceived as wanting to search for an object without reference to its surroundings: "Background is not a significant part of what I want, but is a significant part of the selection process". One museum textiles curator refused to specify a background colour: "I only know the colour of the dress!"

- Use of a relatively abstract drawing tool (using rectangular blocks) has advantages in overcoming the users' own perception of their artistic ability. Most users learned how to use the tool fairly quickly. Users vary in their ability to form a visual abstraction of their search target. The most common expression of this is a tendency to include too much detail, in the form of small blocks. The guidance sheet included a hint to "use big blocks of colour" but, in over half of the evaluations, it was necessary for the evaluator to remind the subject about this. The ability to abstract to larger areas generally improved with experience.

- Users showed remarkably little irritation when their query was unsuccessful, tending instead to blame their own drawing ability.

- Less than half of the users attempted to click within the image area; significantly less than we had anticipated. Of those subjects who clicked within an image area, the majority were seeking further information, typically in

recorded in the query log, although the latter was rarely checked. 
the form of an enlarged image. The remainder wanted to search for similar images.

\subsubsection{User interface}

- Users want to be able to view both the light-box and the drawing tool at the same time, but this can cause problems with window management, especially with smaller screens. Almost without exception, the evaluator gave some assistance in this respect.

- Having a thumbnail image as well as a colour wheel from which to select colours in the drawing tool was found to be useful. Some users requested additional facilities, such as other shapes, and the ability to copy colours from one block to another. On the other hand, a few users felt that the tool was adequately suited to their ability to draw abstract pictures.

- The drawing 'canvas' is square, whereas the images are in either portrait or landscape format. Users varied in their ability:

(a) to comprehend the aspect ratio as important; and

(b) to 'stretch' their drawing to accommodate the aspect ratio.

- The majority of users were comfortable with the action buttons beneath the thumbnail images, although some comments were made about the manner of their implementation. Some users also desired other functions. These included the ability to browse a random selection of images, and a history or bookmark facility.

\subsection{Interpretation}

- User expectations depend on their previous experience, which supports the view that the user interface needs to be flexible to accommodate that range of experience. The observation that most users learned quickly - both in terms of using the system, and also in understanding what CBIR can and cannot achieve - suggests that our interface, and the help provided, are appropriate for the task.

- CBIR queries can be placed in two principal categories: browsing the collection; and searching for specific images. We observed both forms of query. We had anticipated that searches would involve a mixture of drawing and querying for similar images but, in practice, user behaviour tended to concentrate on refinement of the drawing. A small number of subjects were invited to create their own target, as a 'mental image', once they had some experience of the nature of the images in the collection. For these latter cases, there was more evidence of the two search methods being used in conjunction. This may reveal a flaw in the experiment, in that providing a clear target gives too much encouragement to the subject to concentrate on refining the drawing. The behaviour shift when the target is a 'mental image' would tend to support this.

- The images we catalogued for this experiment were predominantly specimen images, defined in section 3.1 as "those images in which the significant object or objects is or are depicted against a neutral background". Having to define a background was of concern to most users and some questioned whether CBIR was really suitable for this type of image. Two users expressed the view that images which did not have such a background would be more appropriate. Our collection did include a limited number of 'general' images, such as paintings and fabric samples. Had our collection mainly comprised such images, we might have obtained some different responses.

\section{Conclusions}

We had an opportunity, as part of ELISE, to carry out experiments into user expectations for content-based image retrieval, and to explore user interface issues. The potential user population is diverse and the ELISE collections offer a broad variety of image types, but constraints of time and funding necessarily limited the scope of our experiments.

We concentrated on how our users reacted to the CBIR interface: what they felt comfortable with, and what left them unsure. Although this was a small experiment, we believe it complements the other studies now underway, and will lead to a better understanding of how practical applications of CBIR can evolve to meet the challenge of image retrieval. 


\section{Acknowledgements}

We gratefully acknowledge stimulating discussions with members of the ELISE consortium. We thank Xiaoming Zhu at the IBM Almaden Research Center for making available to us the source code for the QBIC Java GUI. We also acknowledge the financial support given by the European Commission to the ELISE project.

\section{$7 \quad$ References}

1. Flickner M, Sawnhey H, Niblack W et al. Query by Image and Video Content: The QBIC System, IEEE Computer 1995; 28:23-30 (http://wwwqbic.almaden.ibm.com/ qbic/)

2. Bach J, Fuller C, Gupta A et al. The Virage Image Search Engine: An open framework for image management, SPIE Proceedings 1996; 2670:76-87 (http://www.virage.com/online)

3. Columbia University WebSeek (http://www.ctr.columbia.edu/webseek)

4. Hirate K, Mukherjea S, Okamura Y et al. Object-based navigation: An Intuitive Navigation Style for Contentoriented Integration Environment, Hypertext 97, Southampton, UK 1997; 75-86 (http://www.ccrl.com/amore/)

5. Venters C, Eakins P, Hartley R. The User Interface \& Content-Based Image Retrieval Systems, Information Retrieval Research, Proc. 19 ${ }^{\text {th }}$ Annual BCS IRSG Colloquium on IR Research 1997

6. Bird C, Elliott P. Search Techniques Within a Multiple Database Environment, The Challenge of Image Retrieval, Newcastle 1998 (in press)

7. Niblack W, Zhu X, Hafner J et al. Updates to the QBIC System, SPIE Proceedings 1997; 3312:150-161

8. Gupta A, Jain R. Visual Information Retrieval, Communications of the ACM 1997; 40:71-79

9. Minka T, Picard R. Interactive Learning with a 'Society of Models', Pattern Recognition 1997; 30:565-581

10. Cox I, Miller M, Omohundro S, Yianilos P. PicHunter: Bayesian Relevance Feedback for Image Retrieval, Proc. $13^{\text {th }}$ Intl. Conf. On Pattern Recognition 1996; 361-369

11. Wood M, Campbell N, Thomas B. Searching Large Image Databases using Radial-Basis Function Neural Networks, IEE IPA97 Proceedings 1997; 116-120

12. Kageyama Y, Saito H. Image Retrieval System Capable of Learning the User's Sensibility Using Neural Networks, IEEE Intl. Conf. On Neural Networks 1997; 1563-1567

13. Psarrou A, Konstantinou V, Morse P, O'Reilly P. Content-based search in medieval manuscripts, IEEE Tencon'97, Speech and Image Technologies for Computing and Telecommunications, Brisbane 1997; 187-190

14. Grout C. From 'Virtual Librarian' to Virtual Curator': What is the potential for the emergent Image Recognition Technologies in the Art-Historical Domain?, in Electronic Imaging and the Visual Arts, London 1996 